\title{
Development of Single Camera Finger Touch Position Coordinate Transform Algorithm
}

\author{
Shenjing Chen ${ }^{\text {a, }}$, Lifeng Zhang ${ }^{\text {b }}$, Seiichi Serikawa ${ }^{a}$ \\ ${ }^{a}$ Department of Electrical Engineering and Electronics, Kyushu Institute of Technology, Fukuoka, Japan \\ *n349419s@mail.kyutech.jp
}

\begin{abstract}
This paper has focused on Image processing-based touch sensing systems using new methods. Image processing based single camera figure touch position detection approach has several significant advantages: (1) this method need not extra sensing instruments on the surface or under side of touch screen like resistive membrane-based systems and capacitive-based sensing systems. (2) this approach can convert non-touch screen into touch screen by only attaching cameras on the screen. (3) this approach enables various operations not only by touch but also by gesture operations. The most characteristic point of our system is using only single camera. Using a single view camera, we cannot get the depth information from a single view image. To solve this problem, we use the reflected finger image appears on the back of the screen. Detecting the fingertip and the reflected image on the screen effectively, we enable to detect touch position only using single camera. In this paper, we provide concrete method, algorithm, implement details, and several experimental results.
\end{abstract}

Keywords: Finger detection, Finger tracking, Image processing, Computer vision, Touch panel.

\section{Introduction}

This paper has focused on Image processing-based touch sensing systems. Touch sensing system provides more usable and convenience touches action by allowing user directly operation through finger than traditional interfaces such as keyboard or mouse. For this reason public equipment with large screen such as digital signage and vending machines are increasing these days.

The major touch sensing methods are resistive membrane-based system and capacitive-based system. Resistive membrane-based system is cheap to implement, but it is easy to damage and hard to provide sufficient high sensing accuracy. Therefore, membrane-based touch sensing system is difficult to support more sophisticated or complex user operations. Capacitive-based system can provide more accurate sensing result. But Capacitive-based system requires some extra sensing attachments under the screen [1].

For these reason, Image processing-based touch sensing system is collecting a lot of attention these days. Image processing-based touch sensing system is used to large touch screen for TV weather forecast, Digital Signage. Image processing-based touch sensing systems has advantages: (1) this method need not extra sensing instruments on the surface or under side of touch screen like resistive membrane-based systems and capacitive-based sensing systems. (2) this approach can convert non-touch screen into touch screen by only attaching cameras on the screen. (3) this approach enables various operations not only by touch but also by gesture operations.

However, most developed image processing-based touch sensing system are using a multi cameras to detect the position of the finger by using the principle of triangular surveying.

Therefore, existing image processing-based method has some weak points: (1) Distance is required between the cameras in order to detect the finger. (2) it is necessary to adjust the optical axis precisely of multiple cameras.

Moreover, it is not possible to determine the depth (distance between finger and the screen) using the principle of triangular surveying. From this reason, strictly speaking, the triangular surveying based image processing based system cannot recognize the touch operation. 
Therefore, we propose the Single Camera Finger Touch Position Detection System. The most characteristic point of our system is using only single camera. Using a single view camera to detect the touch action and touch position is a challenging task. Because general speaking, we cannot get the depth information from a single view image.

To solve this problem, we use the reflected finger image appears on the back of the screen. Detecting the fingertip and the reflected image on the screen effectively, we enable to detect touch position only using single camera. Image processing based single camera figure touch position detection approach has several significant advantages: (1) No sensing devices need to be instrumented on the surface of the touch screen. (2) Minimum sensor construction can reduce the failure rate to realize maintenance free system. (3) This approach enables an easy installation and a low-cost touch sensing.

In this paper, chapter 2 describe the proposed system, and fingertip coordinate transform algorithm which calculate the position of the finger exactly, chapter 3 shows the evaluation experiment of fingertip coordinate transform algorithm. Finally, the conclusion and future work is mentioned in chapter 4 .

\section{Proposed System}

\subsection{System Organization}

Figure.1 shows the concept of proposed system. A single camera is fixed at the top of the screen. From the camera image, recognize the fingertip to get $\mathrm{x}$-coordinate and $y$-coordinate and recognize fingertip and reflected image to get z-coordinate. If coordinates of fingertip (blue frame in Figure.1) and reflected image (green frame in Figure.1) overlapping, determine finger has touched the screen.

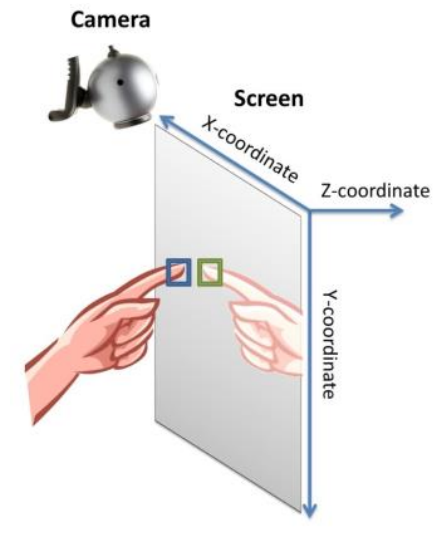

Fig. 1 Concept of proposed system
Figure. 2 shows process flow of our proposed system. Our system performs process as follows:

1. Capture a single view image includes fingertip and reflected image from the camera fixed at the top of the screen.

2. From the capture image, recognize fingertip and reflected image using Haar-like features and Adaboost learning algorithm.

3. Get fingertip and reflected image coordinates.

4. From two obtained coordinates, determine overlap of two coordinates.

5. If coordinates of fingertip and reflected image coordinates overlapping, determine finger has touched the screen.

6. After detecting fingertip, convert the coordinate of fingertip on the camera into the coordinate on the screen.

7. Output the result.

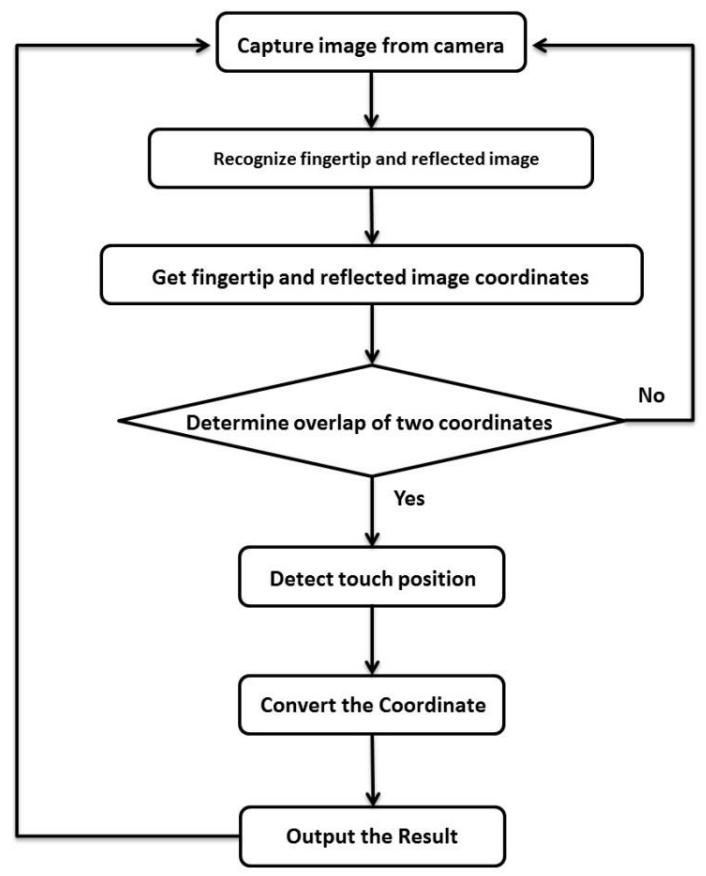

Fig. 2 Process flow

\subsection{Fingertip Coordinate Transform Algorithm}

Because the screen looks trapezoidal shape in camera image. For this reason, we have to convert the coordinate of fingertip on the camera into the coordinate on the screen after detecting fingertip. Figure. 3 shows the image of coordinate transformation. The method of fingertip coordinates transform algorithm based on the idea of projective transformation. $(\mathrm{k}, \mathrm{Nx}, \mathrm{Ny})$ is calibration parameters which add to projection transformation. Normal projective transformation cannot correspond to the noise 
and the camera gap. Therefore, Parameter k works as scale parameter and Parameter Nx,Ny works as sift parameter.

Converted fingertip coordinate $X^{\prime}$ and $Y^{\prime}$ can be computed by Equation (1) by original fingertip coordinate $\mathrm{x}, \mathrm{y}$, conversion parameters $\mathrm{a}, \mathrm{b}, \mathrm{c}, \mathrm{d}, \mathrm{e}, \mathrm{f}, \mathrm{g}, \mathrm{h}$ and calibration parameters $\mathrm{k}, \mathrm{Nx}, \mathrm{Ny}$.

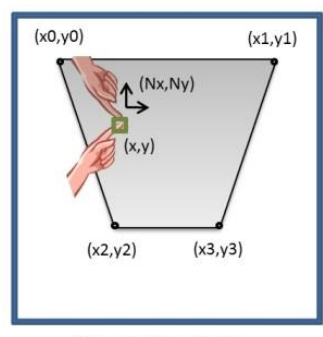

Camera Coordinate

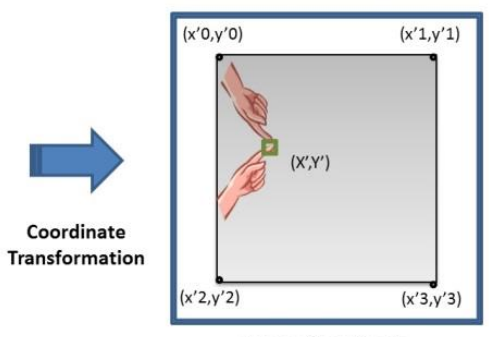

Screen Coordinate
Fig. 3 Image of coordinate transformation

$$
\begin{aligned}
& X^{\prime}=k \frac{a x+b y+c}{g x+h y+1}+N x \\
& Y^{\prime}=k \frac{d x+e y+f}{g x+h y+1}+N y
\end{aligned}
$$

Converted fingertip coordinate $\mathrm{X}^{\prime}, \mathrm{Y}^{\prime}$ can be expressed as a matrix as following Equation (2).

$$
\begin{aligned}
& \left(\begin{array}{c}
X^{\prime} \\
Y^{\prime} \\
1
\end{array}\right)=k\left(\begin{array}{lll}
a & b & c \\
d & e & f \\
g & h & 1
\end{array}\right)\left(\begin{array}{l}
x \\
y \\
1
\end{array}\right)+\left(\begin{array}{c}
N x \\
N y \\
1
\end{array}\right) \\
& =k\left(\begin{array}{ccc}
a+g N y & b+h N x & c+N x \\
d+g N y & e+h N y & f+N y \\
g & h & 1
\end{array}\right)\left(\begin{array}{l}
x \\
y \\
1
\end{array}\right)
\end{aligned}
$$

Conversion parameters a, b, c, d, e, f, g, h can be computed by 8 known points (x0, y0) (x1, y1) (x2, y2) (x3, y3) (x’0, y’0) (x'1, y'1) (x'2, y'2) (x’3, y’3) by following Equation (3). Revision parameters $\mathrm{k}, \mathrm{Nx}, \mathrm{Ny}$ are set by manual .

$$
\left(\begin{array}{l}
a \\
b \\
c \\
d \\
e \\
f \\
g \\
h
\end{array}\right)=\left(\begin{array}{ccccc}
x 0 & y 0 & 1 & -x^{\prime} 0 x 0 & -y^{\prime} 0 y 0 \\
x 1 & y 1 & 1 & -x^{\prime} 1 x 1 & -y^{\prime} 1 y 1 \\
x 2 & y 2 & 1 & -x^{\prime} 2 x 2 & -y^{\prime} 2 y 2 \\
x 3 & y 3 & 1 & -x^{\prime} 3 x 3 & -y^{\prime} 3 y 3 \\
x 0 & y 0 & 1 & -x^{\prime} 0 y 0 & -y^{\prime} 0 y 0 \\
x 1 & y 1 & 1 & -x^{\prime} 1 y 1 & -y^{\prime} 1 y 1 \\
x 2 & y 2 & 1 & -x^{\prime} 2 y 2 & -y^{\prime} 2 y 2 \\
x 3 & y 3 & 1 & -x^{\prime} 3 y 3 & -y^{\prime} 3 y 3
\end{array}\right)^{-1}\left(\begin{array}{l}
x^{\prime} 0 \\
x^{\prime} 1 \\
x^{\prime} 2 \\
x^{\prime} 3 \\
y^{\prime} 0 \\
y^{\prime} 1 \\
y^{\prime} 2 \\
y^{\prime} 3
\end{array}\right)
$$

\section{Evaluation Experiment}

In order to confirm the speed and accuracy of our coordinate transform algorithm, we conducted an evaluation experiment and created an experimental program using C++ on Visual Studio 2012. The experimental environment is by using web camera to capture $320 \times 240$ pixel image, OS is Windows7, CPU is Core-i3 $2.93 \mathrm{GHz}$ and Memory is $2 \mathrm{~GB}$.

We attached a web camera at the top of the tablet like Figure.4.

Figure.5 shows the original captured image form web camera.

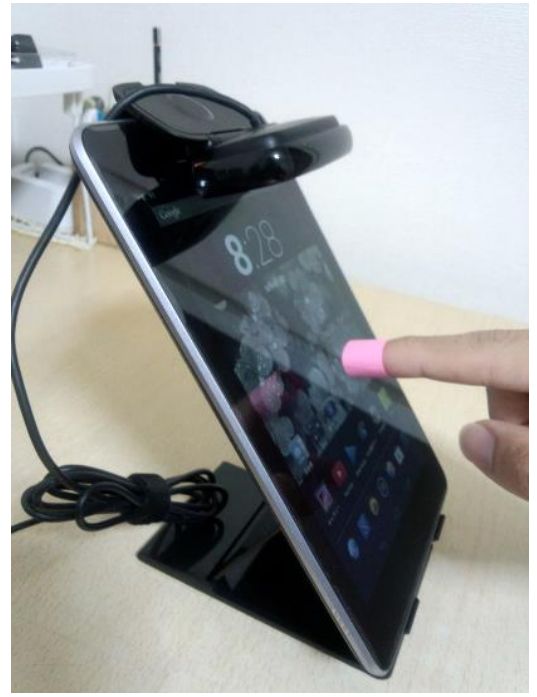

Fig.4 The state that attaches a web camera to the tablet

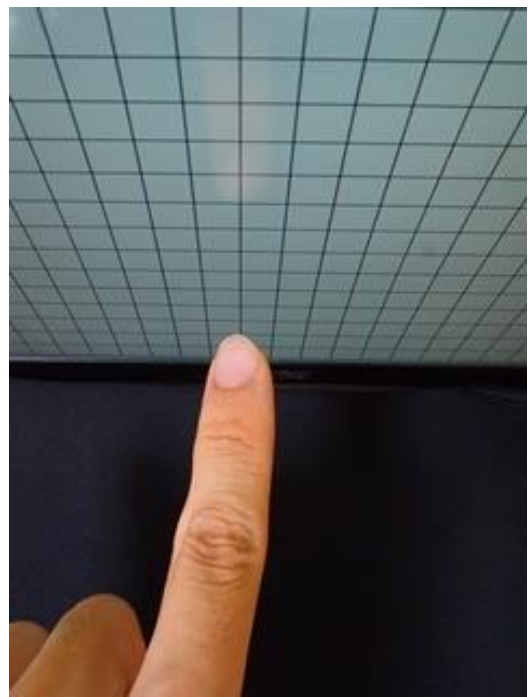

Fig.5. Original captured image 


\subsection{Transformation Speed Experiment Result}

Table 1 shows Resolution and Calculation time of our fingertip coordinate transform algorithm.

Table 1 Resolution and Calculation time of our fingertip coordinate transform algorithm

\begin{tabular}{|l|l|}
\hline Resolution & Calculation Time \\
\hline $320 \times 240$ pixel & $0.12 \sim 0.14 \mathrm{~ms}$ \\
\hline
\end{tabular}

Our coordinate transform algorithm calculates very fast from coordinate transformation experiment result.

\subsection{Transformation Accuracy Experiment Result}

Figure. 6 shows the result of our coordinate transform algorithm from original captured image (Figure.5).

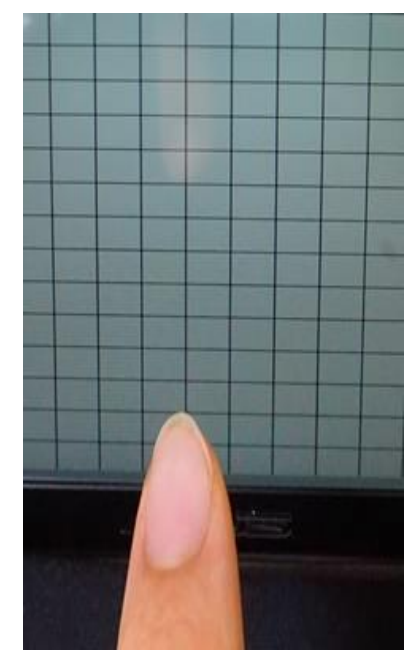

Fig.6 result of coordinate transform algorithm

Due to our system using projection transform algorithm, spatial resolution will decrease at the bottom side.

Assuming an angle $\theta$ between the upper side and aspect side shows in Figure.7, spatial resolution will decrease as Equation (4).

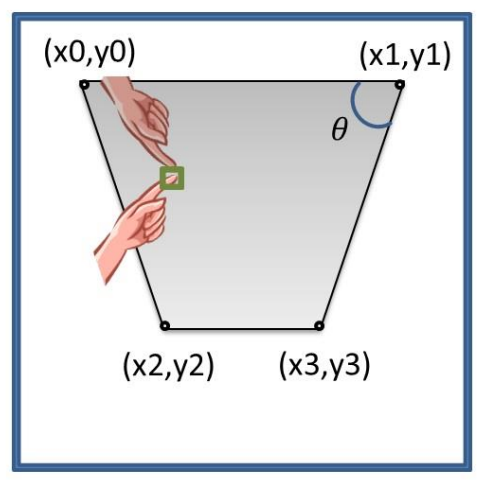

Fig.7 Image of angle

$$
\frac{\left(x_{1}-x_{0}\right)-\frac{2\left(y_{2}-y_{0}\right)}{\tan \theta}}{\left(x_{1}-x_{0}\right)}
$$

Figure. 8 shows the calculate result of reduction rate of the spatial resolution.

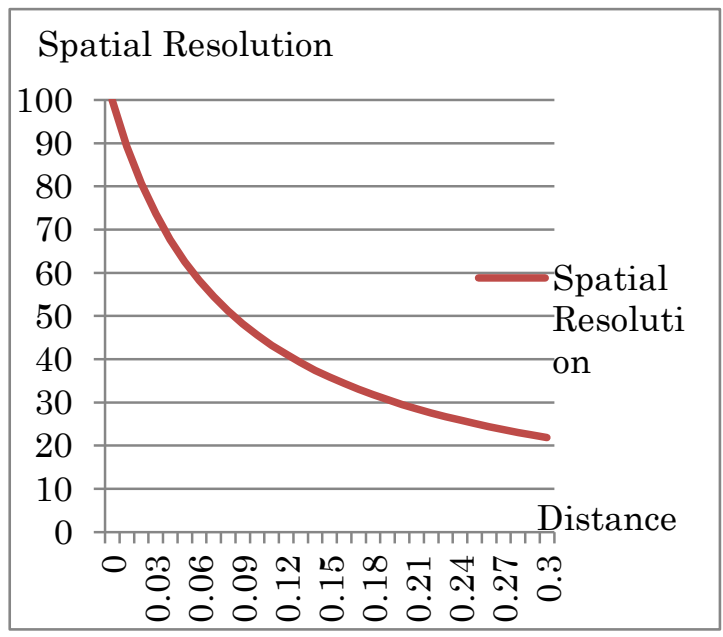

Fig.8 Reduction rate of the spatial resolution

\section{Conclusions}

In this paper, we proposed a Finger Touch Detection and Position Detection System using a Single Camera. The coordinate transformation experiment result shows that our coordinate transform algorithm calculates very fast in real time, and converts the coordinate of fingertip on the camera image into the coordinate on the screen correctly. However, the unstable of finger coordinate transformation is a problem had not been solved. As a future work, we would like to devise a correction algorithm to get improved transformation accuracy and processing time.

\section{References}

(1) Chen, Y.-L.; Liang, W.-Y.; Chiang, C.-Y.; Hsieh, T.-J.; Lee, D.-C.; Yuan, S.-M.; Chang, Y.-L. "Vision-based finger detection, tracking, and event identification techniques for multi-touch sensing and displaying systems". Sensors 2011, 11, 6868-6892.

(2) Q. Chen, N. D. Georganas, and E. M. Petriu, "Hand gesture recognition using Haar-like features and a stochastic context-free grammar," IEEE Trans. Instrum. Meas., vol. 57, no. 8, pp. 1562-1571, Aug. 2008.

(3) S. K. Kang, M. Y. Nam and P. Rhee, "Color Based Hand and Finger Detection Technology for User Interaction,'Proc.IEEE Int'l Conf. Convergence and Hybrid Information Technology, pp. 229-236, 2008.

(4) Bhuyan, M. K., Debanga Raj Neog, and Mithun Kumar Kar. "Fingertip detection for Hand pose recognition." 
International Journal of Computer Science and Engineering (IJCSE) 4.3 (2012).

(5) Lienhart, Rainer, and Jochen Maydt. "An extended set of haar-like features for rapid object detection." Image Processing. 2002. Proceedings. 2002 International Conference on. Vol. 1. IEEE, 2002.

(6) Messom, Chris, and Andre Barczak. "Fast and efficient rotated haar-like features using rotated integral images." Australasian Conference on Robotics and Automation. Vol. 1. 2006. 